\title{
BMJ Open Point-of-care washing of allogeneic red blood cells for the prevention of transfusion-related respiratory complications (WAR-PRC): a protocol for a multicenter randomised clinical trial in patients undergoing cardiac surgery
}

\author{
Matthew A Warner, ${ }^{1}$ Ian J Welsby, ${ }^{2}$ Phillip J Norris, ${ }^{3}$ Christopher C Silliman, ${ }^{4}$ \\ Sarah Armour, ${ }^{5}$ Erica D Wittwer, ${ }^{5}$ Paula J Santrach, ${ }^{6}$ Laurie A Meade, ${ }^{5}$ \\ Lavonne M Liedl, ${ }^{5,7}$ Chelsea M Nieuwenkamp, ${ }^{6}$ Brian Douthit, ${ }^{8}$ \\ Camille M van Buskirk, ${ }^{9}$ Phillip J Schulte, ${ }^{7}$ Rickey E Carter, ${ }^{7}$ Daryl J Kor ${ }^{5}$
}

To cite: Warner MA, Welsby IJ, Norris PJ, et al. Point-of-care washing of allogeneic red blood cells for the prevention of transfusion-related respiratory complications (WAR-PRC): a protocol for a multicenter randomised clinical trial in patients undergoing cardiac surgery. BMJ Open 2017;7:e016398. doi:10.1136/ bmjopen-2017-016398

- Prepublication history and additional material for this paper are available online. To view these files please visit the journal online (http://dx.doi. org/10.1136/bmjopen-2017016398).

Received 21 February 2017 Revised 8 May 2017 Accepted 15 June 2017

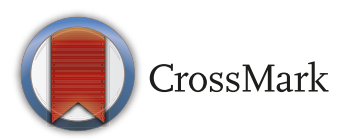

For numbered affiliations see end of article.

Correspondence to

Dr Daryl J Kor;

kor.daryl@mayo.edu

\section{ABSTRACT}

Introduction The transfusion-related respiratory complications, transfusion-related acute lung injury (TRALI) and transfusion-associated circulatory overload (TACO), are leading causes of transfusion-related morbidity and mortality. At present, there are no effective preventive strategies with red blood cell (RBC) transfusion. Although mechanisms remain incompletely defined, soluble biological response modifiers (BRMs) within the RBC storage solution may play an important role. Point-of-care (POC) washing of allogeneic RBCs may remove these BRMs, thereby mitigating their impact on post-transfusion respiratory complications.

Methods and analysis This is a multicenter randomised clinical trial of standard allogeneic versus washed allogeneic RBC transfusion for adult patients undergoing cardiac surgery testing the hypothesis that POC RBC washing is feasible, safe, and efficacious and will reduce recipient immune and physiologic responses associated with transfusion-related respiratory complications. Relevant clinical outcomes will also be assessed. This investigation will enrol 170 patients at two hospitals in the USA. Simon's two-stage design will be used to assess the feasibility of POC RBC washing. The primary safety outcomes will be assessed using Wilcoxon Rank-Sum tests for continuous variables and Pearson chi-square test for categorical variables. Standard mixed modelling practices will be employed to test for changes in biomarkers of lung injury following transfusion. Linear regression will assess relationships between randomised group and posttransfusion physiologic measures.

Ethics and dissemination Safety oversight will be conducted under the direction of an independent Data and Safety Monitoring Board (DSMB). Approval of the protocol was obtained by the DSMB as well as the institutional review boards at each institution prior to enrolling the first study participant. This study aims to provide important information regarding the feasibility of POC washing of allogeneic RBCs and its potential impact on ameliorating post-transfusion respiratory complications. Additionally, it will inform the feasibility and scientific merit of pursuing a more definitive phase II/III clinical trial.

Registration ClinicalTrials.gov registration number is NCT02094118 (Pre-results).

\section{INTRODUCTION}

Transfusion-related pulmonary complications, including transfusion-related acute lung injury (TRALI) and transfusion-associated circulatory overload (TACO), are the leading cause of serious transfusion-related adverse events. TRALI is the primary cause of transfusion-related death and, although seemingly less appreciated, TACO has been the second leading cause of transfusion-related death in recent years. In addition to their associated mortality, both syndromes result in substantial resource utilisation and associated healthcare cost. A large proportion of patients who develop TRALI will require intensive care unit (ICU) admission and ventilator support. ${ }^{12}$ Similarly, up to $21 \%$ of TACO cases have been reported as life-threatening and associated with increased lengths of ICU and hospital stays. ${ }^{3-6}$ Although specific preventative strategies have dramatically reduced the incidence of plasma-associated TRALI (eg, male-only plasma donation), no prevention strategies exist for red blood 


\section{Strengths and limitations of this study}

- Significant knowledge gap, specifically understanding whether point-of-care washing of allogeneic red blood cells (RBCs) is safe, feasible, and efficacious in ameliorating recipient immune and physiologic responses to transfusion that are associated with transfusion-related respiratory complications

- In addition to exploring immune and physiologic response, the trial is also designed to explore clinical outcomes in order to inform the merit and feasibility of future phase I//III clinical trials

- Large and accessible at-risk population

- Established multicenter clinical trial infrastructure

- Detailed and measured statistical approach

- Multidisciplinary expertise in translational, patient-centred transfusion research

- Potential for substantial clinical impact should the intervention prove safe and effective

- Unproven feasibility of point-of-care washing in a time-sensitive environment

- Candidate biomarkers for transfusion-related lung injury may not fully represent or capture true causal pathways

- The inflammatory response accompanying cardiac surgery may mask between-group differences in the immune and physiologic responses to transfusion therapies

- Inconsistent timing and dose of red blood cell transfusion

- Study will test the impact of modifying the RBC storage solution with POC washing, but will not clarify the impact of storage on the RBCs themselves

- Unclear effects of RBC storage duration

Study not adequately powered for clinical outcomes

cell (RBC)-associated TRALI or TACO. Indeed, the lack of safe and feasible strategies that can mitigate risk of RBC-associated TRALI and TACO represent critical knowledge gaps in transfusion medicine.

While TRALI and TACO share a similar clinical phenotype of pulmonary oedema and hypoxemic respiratory insufficiency, each is believed to result from distinct pathologic processes. ${ }^{35-9}$ TRALI is believed the result of a two-hit process beginning with pulmonary endothelial activation resulting in leucocyte priming, sequestration, and activation followed by endothelial injury with inflammatory lung oedema. The first insult typically relates to recipient factors (eg, surgery, trauma, infection) and the second 'hit' from the infusion of mediators in the blood component. For high-plasma volume components including plasma or apheresis platelets, this is believed most often the result of donor anti-leukocyte antibodies reacting with recipient cognate antigens. In contrast, multiple lines of evidence suggest alternate mechanisms are at play with RBC-associated TRALI. ${ }^{70-12}$ Here, the second insult is generally attributed to the infusion of soluble biological response modifiers (BRMs) residing in the RBC supernatant.

Conversely, TACO has classically been attributed to fluid overload in the setting of transfusion. However, a large proportion of reported TACO cases present after a single blood unit exposure without overt signs of systemic volume overload. ${ }^{1314}$ Moreover, TACO is characteristically accompanied by a marked hypertensive response that exceeds what would be expected from a volume challenge alone, suggesting the potential presence of vasoactive substances in the transfused product that may increase systemic vascular resistance. ${ }^{15-17}$ An abrupt increase in systemic vascular resistance may result in increased cardiac filling pressures, thereby increasing risk for hydrostatic pulmonary oedema. Hence, it is possible that additional and potentially synergistic pathophysiologic processes are at play in TACO. Indeed, a growing body of evidence suggests that BRMs contained within the supernatant of stored RBC (eg, free haemoglobin, RBC microparticles) may act on vascular smooth muscle tone and contribute to TACO. ${ }^{18-23}$

Washing of allogeneic RBCs can remove soluble contaminants in the RBC supernatant including chemokines, biologically active lipids, cellular debris, microaggregates, and other BRMs. ${ }^{24-27}$ Interestingly, a large investigation noted a complete absence of reported TRALI and TACO cases following transfusion of more than 28000 units of washed allogeneic RBCs. ${ }^{28}$ Additionally, RBC washing has been associated with decreased adverse immunologic effects in transfused trauma patients and improved survival in transfusion recipients with acute leukaemia. ${ }^{2930}$ Although promising, washing stored RBCs has been largely discounted due to concerns related to cost and feasibility. ${ }^{31}$ However, as there are no effective prevention strategies for RBC-associated TRALI or TACO, and there is clear biologic plausibility for cause-effect relationships between the infusion of soluble BRMs and the development of life-threatening transfusion-related respiratory complications, further investigation is clearly warranted.

To enhance our understanding regarding the role of point-of-care allogeneic RBC-washing as a means to mitigate transfusion-related respiratory complications, the Washing of Allogeneic Red blood cells for the Prevention of Respiratory Complications (WAR-PRC) Study was developed. This is a multicenter randomised clinical trial, supported by the National Institutes of Health-National Heart, Lung and Blood Institute (NIH grant number: R01 HL121232, PIs: Drs. Kor, Welsby). The trial aims to test the feasibility, safety, and efficacy of point-of-care RBC washing using an FDA-approved autotransfusion device known as the Continuous Autotransfusion System (CATS) in adult cardiac surgery patients receiving allogeneic RBC transfusion. Cardiac surgical patients were selected as the target population given the frequency of large-volume RBC transfusion in this practice location, ${ }^{32}$ the well-described risks of postoperative respiratory complications in this patient population, ${ }^{33-36}$ and the presence and routine use of the cell washing strategies (auto-transfusion) in this environment. This paper describes the study procedures and planned analyses for this clinical trial. 


\begin{tabular}{l}
\hline Inclusion Criteria \\
\hline - Cardiac surgery \\
- Age $\geq 18$ years \\
- Predicted RBC \\
requirement $\geq 4$ \\
units
\end{tabular}

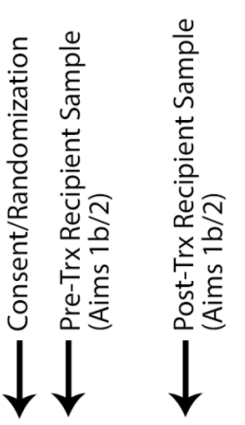

Intraoperative

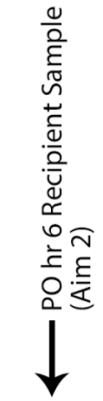

POD 0 (Day of Surgery)

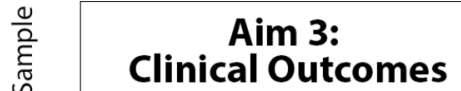

Intervention: CATS-washed versus standard-issue RBCs

\begin{tabular}{|c|c|}
\hline Aim 1: Feasibility, Safety, and Efficacy of RBC Washing & Physiologic Outcomes \\
\hline $\begin{array}{l}\text { Feasibility of Point-of-Care Washing (Aim 1a) } \\
\text { - Off-protocol transfusions (number, proportion) } \\
\text { - Time to intervention RBC transfusion }\end{array}$ & $\begin{array}{l}\text { Respiratory } \\
\text { assessments: }\end{array}$ \\
\hline $\begin{array}{l}\text { Safety of Point-of-Care Washing (Aim 1b) } \\
\text { - Total Hb, CFH, haptoglobin (RBC recipient) }\end{array}$ & - $\mathrm{PaO} 2 / \mathrm{FiO} 2$ \\
\hline $\begin{array}{l}\text { Efficacy of RBC Washing (Aim 1c) } \\
\text { - Pre/post wash lipids, SCD40L, CCL5, RBC-MP, CFH (RBC component) }\end{array}$ & $\begin{array}{l}\text { - } \mathrm{SpO} 2 / \mathrm{FiO} 2 \\
\text { - Tidal volume }\end{array}$ \\
\hline $\begin{array}{l}\text { Aim 2: Recipient responses to RBC Washing } \\
\text { Intermediate markers of lung injury (Aim 2a - TRALI) } \\
\text { - IL-6, IL-8, PAI-1, RAGE (RBC recipient) }\end{array}$ & $\begin{array}{l}\text { - PEEP } \\
\text { - Peak airway pressure }\end{array}$ \\
\hline $\begin{array}{l}\text { Cardiopulmonary response to } \mathrm{RBC} \text { transfusion (Aim } \mathbf{2 b} \text { - TACO) } \\
\text { - SVR, MAP, PCWP, SpO2, FiO2 (RBC recipient) }\end{array}$ & - Plat. airway pressure \\
\hline
\end{tabular}

BRM relationships with recipient responses to RBC washing (Aim 2c)

- Lipids, sCD40L, CCL5, RBC-MPs, CFH (RBC component, recipient)

- IL-6, IL-8, PAI-1, RAGE and SVR, MAP, PCWP, SpO2, FiO2

Figure 1 Schematic of the planned study procedures. ALI, acute lung injury; CATS, Continuous Autotransfusion System; CCL5, chemokine ligand 5; CFH, cell free haemoglobin; CHF, congestive heart failure; FiO2, fraction of inspired oxygen; Hb, haemoglobin; MAP, mean arterial pressure; PAI-1, plasminogen activator inhibitor 1; PaO2, arterial partial pressure of oxygen; PCWP, pulmonary capillary wedge pressure; PEEP, positive end expiratory pressure; PO, postoperative; POD, postoperative day; RAGE, receptor of advanced glycation end-products; RBC, red blood cell; RBC-MP, red blood cell microparticle; Rxs, reactions; sCD40L, soluble CD40 ligand; SOFA, sequential organ failure assessment; SpO2, oxygen saturation by pulse oximetry; SVR, systemic vascular resistance; TACO, transfusion-associated circulatory overload; TRALI, transfusion-related acute lung injury; Trx, transfusion.

\section{METHODS AND ANALYSIS \\ Study design}

To test the hypothesis that point of care (POC) allogeneic RBC-washing will be safe, feasible, and associated with amelioration of intermediate markers of TRALI and TACO, a multi-centre, single-blinded (outcome assessor), parallel group, phase I/II randomised clinical trial has been designed. The ClinicalTrials.gov registration number is NCT02094118. An outline of the study design, procedures, and aims is displayed in figure 1 .

\section{Study population}

Adult patients aged 18 years and older undergoing cardiac surgery with heightened risk for large volume RBC transfusion, defined as a predicted RBC transfusion requirement of greater than or equal to four units, will be enrolled. To facilitate the identification of patients at high risk for RBC transfusion, a validated cardiac surgery prediction model will be utilised. ${ }^{37}$ A cut-off of 4 predicted units of RBC administration was chosen because this transfusion volume is associated with increased risk of pulmonary complications following cardiac surgery. ${ }^{38}$ Additionally, it has been identified as a common 'RBC dose' implicated in patients with TRALI and/or TACO. ${ }^{8}{ }^{39}$ This threshold will also still identify a sizeable cardiac surgery population, ensuring study feasibility. A complete list of exclusion criteria including the justification for each is shown in table 1.

\section{Interventions}

Study intervention: The intervention in this investigation will be implemented for all allogeneic RBCs administered on the day of sugery, including intraoperative and postoperative transfusions. The decision to administer an allogeneic RBC transfusion will be left to the responsible clinical service and will not be prespecified in the 
Table 1 Study exclusion criteria

\begin{tabular}{ll}
\hline Exclusion criteria & Justification \\
\hline Emergency surgery & Inability to randomise/perform study procedures \\
\hline IgA deficiency & Not ethical to randomise to standard issue RBCs \\
$\begin{array}{l}\text { History of severe recurrent transfusion reaction } \\
\text { Refusal to receive allogeneic RBCs }\end{array}$ & Not ethical to randomise to standard issue RBCs \\
\hline Refusal to provide informed consent & Not ethical to enrol into trial \\
\hline Prevalent acute lung injury prior to randomization & Inability to adequately assess outcome \\
\hline Prevalent hydrostatic pulmonary oedema prior to randomization & Inability to adequately assess outcome \\
\hline Expected hospital stay<48hours & Incomplete study procedures and outcome data \\
Not anticipated to survive>48hours & Incomplete study procedures and outcome data \\
\hline Previously enrolled in this trial & Violation of the independence assumption \\
\hline $\begin{array}{l}\text { Pulmonary artery catheter placement not planned for the surgical } \\
\text { procedure }\end{array}$ & Inability to assess key physiologic parameters outlined in \\
Use of home oxygen therapy & the study protocol \\
Complex RBC antibody profiles & Inability to assess oxygen use outcome \\
Need for the use of irradiated RBCs & Washing not feasible due to testing delays \\
\hline
\end{tabular}

Patients will be recruited and enrolled at two academic medical centres in the USA (Mayo Clinic, Rochester, MN; Duke University Medical Centre, Raleigh, NC) with substantial experience in RBC-washing and transfusion management for cardiac surgery. With regards to type of cardiac surgery, study coordinators will screen all adult patients scheduled to undergo coronary artery bypass grafting (CABG) surgery, complex cardiac valve surgery, pericardial resection, and/or ascending aortic surgery in one of the two participating institutions. Eligible patients will be approached before their elective surgical procedure by a member of the study team for informed consent. A study identification (ID) number will be assigned to each study participant and randomization will occur after receipt of informed consent, but before entry to the operating room for the scheduled procedure. Screening logs will be maintained at each site to allow generation of a CONSORT diagram.

study protocol. There are several reasons for this lack of prespecified indications for RBC transfusion. First, the target population frequently experiences major acute blood loss. During these circumstances, typical measures assessing the need for RBC transfusion, such as threshold haemoglobin or hematocrit values, do not reflect true RBC cell mass nor the need for RBC transfusion. Moreover, the process of obtaining these laboratory results may be associated with unacceptable time delays when bleeding is severe. Additionally, this design facilitates a more meaningful understanding of the feasibility of RBC washing in clinical practice.

When a clinical decision to proceed with allogeneic $\mathrm{RBC}$ transfusion has been made, the RBC product will be immediately prepared in the operating room (or in the ICU room if administered postoperatively) according to the allocated treatment assignment (washed versus standard issue). For patients randomised to the control group arm, all RBCs administered on the day of surgery will be standard-issue allogeneic RBCs. Detailed RBC unit characteristics including the type of RBC product (ie, whole-blood derived versus apheresis), processing, and additive characteristics for each clinical site are provided (online Supplementary material 1). For patients randomised to the intervention arm of this trial, all allogeneic RBCs administered on the day of surgery will be washed with the CATS device prior to transfusion. RBC washing may occur on allogenic RBCs of any storage duration until the time of expiration, and washed units may be stored for up to 24 hours if not immediately administered. The CATS device was chosen over more traditional cell washing machines (eg, the Cobe 2991 Cell Processor) due to the reduced time needed for cell washing with CATS as well as the reduced risk for hemolysis with the CATS device. ${ }^{40}$ As previously described and confirmed in our preliminary data, pre-dilution of stored, allogeneic RBCs results in the most effective elimination of supernatant. ${ }^{26}$ Therefore, a 4:1 dilution consisting of $1200 \mathrm{~mL}$ saline to $300 \mathrm{~mL}$ RBCs will be added to the reservoir of the CATS by gravity drainage. The 'high quality' wash mode option will be selected for processing. ${ }^{29}$ Washed RBCs will then be drained from the reinfusion reservoir into sterile transfer bags (Fenwal Inc, Lake Zurich, IL) for transfusion. A full description of the standard operating procedures for RBC washing are available (online Supplementary material 2). Of note, all allogeneic RBC units at the two participating institutions undergo pre-storage leucocyte reduction, although differences in the exact timing of this intervention exist between the two sites for whole-blood derived RBCs (online Supplementary material 1).

\section{Off-protocol transfusions}

In the setting of cardiac surgery, it is occasionally necessary to provide allogeneic RBCs in an emergent fashion (eg, acute, life-threatening bleeding). In this circumstance, time-delays due to study-related activities may prove unsafe. To address this potential scenario, our study protocol will allow the administration of emergency 
'off-protocol' allogeneic RBCs. 'Off-protocol' RBC transfusions will be administered as per standard institutional practice. These RBC transfusion episodes will be specifically noted as 'off-protocol' and will be summarised and analysed to assist in assessing the feasibility of point-of-care RBC washing in patients undergoing cardiac surgery (see statistical description below). In addition, autotransfusion ('cell-saver') is frequently used in this patient population. Cell-salvage will be implemented at the discretion, and under the direction, of the clinical team. If cell-salvage is employed, the device used for this procedure will be distinct and separate from the intervention CATS device.

\section{Co-interventions}

Intraoperative care that is not directly related to this study protocol will be at the discretion of the responsible clinical team(s) (eg, this protocol will not standardise intraoperative anaesthetic care or surgical procedures). However, clinical care decisions that may affect the development of respiratory dysfunction and associated outcomes will be standardised to the greatest extent possible. To this end, the protocol specifies optimal ventilator strategies for both the operating room (OR) and ICU environments, including tidal volumes less than or equal to $8 \mathrm{~mL} / \mathrm{kg}$ predicted body weight, peak inspiratory pressures less than $35 \mathrm{~cm} \mathrm{H}_{2} \mathrm{O}$, and positive end-expiratory pressures (PEEP) equal to or greater than $5 \mathrm{~cm} \mathrm{H}_{2} \mathrm{O}$. Similarly, although RBC transfusion thresholds are not pre-specified in this protocol, restrictive transfusion practices will be advised in the postoperative period with a haemoglobin target greater than $8 \mathrm{~g} / \mathrm{dL}$ in the absence of acute bleeding and/or ischemia. This transfusion threshold was chosen as it is the current standard of care at the two participating institutions. Standardisation of best practices in at-risk patients will decrease the heterogeneity of the risk modifiers that may otherwise confound our associations of interest. Additionally, each centre has adopted protocols on daily spontaneous awakening and spontaneous breathing trials to facilitate standardised weaning from ventilators following cardiac surgery. Non-intubated patients will undergo standard titration of oxygen twice daily (at 0700 and $1900, \pm 2$ hours). Patients saturating $\geq 92 \%$ on room air will not receive supplemental oxygen, unless specifically requested by the primary service. If the primary care service requests oxygen supplementation for a patient saturating $\geq 92 \%$ on room air, the reason for the deviation from oxygen weaning will be documented. Patients will continue to undergo evaluation for oxygen titration until liberation from oxygen therapy for 24 hours, hospital day 28, or hospital discharge, whichever comes first.

Related conditions and variables of interest: Pertinent baseline demographics and clinical characteristics such as age, sex, race, preoperative cardiac function, and comorbidities will be recorded. Additional variables of note will include vital signs and laboratory values that are obtained during the course of routine care, APACHE IV scores, administration of statins, ace-inhibitors, angiotensin-receptor blockers, beta-blockers, diuretics, antiplatelet agents, non-steroidal anti-inflammatory drugs, insulin, amiodarone, or steroids, blood product administration up to day 28 or hospital discharge, whichever comes first, daily fluid status, estimated blood loss, and vasopressor requirements.

\section{Outcomes}

Feasibility, Safety, and Efficacy Outcomes (Study aim 1): The primary feasibility outcome will be the number and proportion of off-protocol allogeneic RBC transfusions administered during the study intervention period (ie, day of surgery). A secondary feasibility outcome will be the time required for the RBC washing procedures defined as the time from determination of allogeneic RBC need by the clinical team to time of delivery of the RBC unit to the clinical team. This time will be computed for all patients and all transfusions during the study intervention period in both the intervention and control cohorts.

The primary safety outcomes include the change in the RBC recipient's haemoglobin concentration from pre- to post-transfusion as well as the concentration of cell-free haemoglobin (CFH) and haptoglobin following RBC transfusion. To assess the primary safety outcomes, samples for total haemoglobin, $\mathrm{CFH}$, and haptoglobin will be obtained prior to transfusion, within $30 \mathrm{~min}$ following the first RBC transfusion, as well as 6 hours $( \pm 30 \mathrm{~min})$ and 18 hours $( \pm 30 \mathrm{~min})$ after the end of the first RBC transfusion for all study participants. Additionally, the number of units and corresponding volume of RBC transfusion will be recorded and compared between groups to evaluate the impact of washing on RBC mass in individual units. Evidence for acute kidney injury defined according to Acute Kidney Injury Network (AKIN) criteria will be assessed throughout hospitalisation as a secondary safety outcome measure. ${ }^{41}$ If the patient remains in the hospital, safety labs will also be drawn on study day number 5 .

The primary outcome evaluating the efficacy of the washing procedures will be the change in the concentration of BRMs including neutral lipids, soluble CD40 ligand (sCD40L), chemokine ligand 5/regulated on activation, normal T-cell expressed and secreted (CCL5/RANTES), RBC microparticles (RBC-MPs), and CFH in the washed RBC component from the pre- to the post-wash phase. These data will allow for calculation of CATS-related elimination rates of BRMs. Blood sampling and biomarker handling procedures have been previously described, ${ }^{42} 43$ and a brief overview of laboratory handling is provided (online Supplementary material 3).

Mechanistic Outcomes (Study aim 2): The concentration of multiple, validated biomarkers representing the primary pathways leading to development of lung injury will be assessed in the study participants. These pre-specified plasma biomarkers are displayed in table 2.

As study participants are expected to receive variable numbers of RBC transfusions at inconsistent times, four discrete time points have been chosen for assessment of these lung injury biomarkers. The first and second 
Table 2 Lung injury biomarkers and exploratory potentially pathogenic biologic response modifiers

\begin{tabular}{lll}
\hline Validated lung injury biomarkers & Primary process evaluated & Supporting Evidence \\
\hline Interleukin-6 & Inflammation & 505960 \\
\hline Interleukin-8 & Inflammation & $455059-61$ \\
\hline Plasma activator inhibitor-1 & Dysregulated coagulation & $5061-64$ \\
\hline von Willebrand Factor & Endothelial injury & $65-70$ \\
\hline sICAM-1 & Endothelial injury & $45596171-73$ \\
Surfactant protein D & Epithelial injury & 45597475 \\
Receptor of advanced glycation end products & Epithelial injury & 457677 \\
\hline Exploratory pathogenic BRMs & Primary process evaluated & Supporting Evidence \\
\hline Neutral lipids & Lung inflammation & 1055 \\
\hline sCD40L & Lung inflammation & 12 \\
\hline CCL5/RANTES & Lung inflammation & 157879 \\
RBC-derived microparticles & NO scavenging & 212380 \\
Cell-free haemoglobin & NO scavenging & 182280 \\
N-terminal brain natriuretic peptide & Ventricular stretch/volume-overload & $81-83$ \\
\hline
\end{tabular}

ICAM-1, intercellular adhesion molecule-1; BRMs, biologic response modifiers; sCD40L, soluble CD40 ligand; CCL5/RANTES, chemokine ligand 5/regulated on activation, normal T-cell expressed and secreted; NO, nitric oxide.

samples will be obtained from the recipient prior to transfusion and within $30 \mathrm{~min}$ following the first intervention or control RBC unit administered. For the third and fourth assessments, samples will be obtained 6 hours $( \pm 30 \mathrm{~min})$ and 18 hours $( \pm 30 \mathrm{~min})$ from the end of the first study RBC transfusion.

Secondary analyses will include detailed assessment of cardiopulmonary responses to RBC transfusions, with specific variables to be assessed shown in table 3 .

Each of these physiologic variables will be assessed and recorded immediately prior to the study $\mathrm{RBC}$ transfusion and again immediately after the transfusion (within $30 \mathrm{~min}$ ). Standard operating procedures for these cardiopulmonary assessments will be defined prior to study onset according to previously established recommendations. ${ }^{44}$ These secondary outcomes will allow a more

Table 3 Physiologic assessments during the study intervention period

\begin{tabular}{ll}
\hline Respiratory Variables & Haemodynamic Variables \\
\hline $\begin{array}{l}\text { Arterial partial pressure of } \\
\text { oxygen }\end{array}$ & Mean arterial pressure \\
Arterial oxygen saturation & Heart rate \\
\hline Fraction of inspired oxygen & Cardiac output \\
Tidal volume & Right atrial pressure \\
\hline Peak airway pressure & $\begin{array}{l}\text { Pulmonary artery wedge } \\
\text { pressure }\end{array}$ \\
\hline $\begin{array}{l}\text { Plateau airway pressure } \\
\text { Positive end-expiratory } \\
\text { pressure }\end{array}$ & Systemic vascular resistance* \\
\hline
\end{tabular}

*Systemic vascular resistance (SVR) will be calculated using the following equation: SVR $\left(\right.$ dyns $\left./ \mathrm{cm}^{5}\right)=[($ Mean arterial pressure right atrial pressure)/cardiac output] x 80 . detailed assessment of the cardiopulmonary response to RBC transfusion and will be expected to provide important insight into the pathophysiology of TACO. Of note, pulmonary artery catheter placement is standard of care for this patient population at both enrolling institutions.

In an attempt to evaluate specific potential mechanistic pathways for TRALI and TACO, exploratory putative BRMs have also been selected (Study aim 2; table 2). In the recipient, samples for the putative BRM assessments will be obtained at the same time points outlined above for the lung injury biomarker samples. To better elucidate the relationship between the dose of these soluble BRMs in the RBC components, their subsequent concentration in the recipient, and their ultimate relationship to the recipient's cardiopulmonary response to transfusion, levels of these potential putative agents will be determined in the RBC components prior to transfusion in both the washed and standard issue cohorts as well as in the transfusion recipient. As enrolled patients are expected to receive four or more units of allogeneic RBCs, samples will be obtained from the RBC component for all RBC units administered up to and including the fourth unit for each study participant. For those in the intervention arm, this will be a post-wash sample. Of note, there exists the potential for incomplete capture of relevant information in those who receive larger volumes of RBC transfusion. However, a four-unit cut-off represents a compromise between study feasibility and scientific validity.

Clinical Outcomes (Study aim 3): To facilitate the design and conduct of future clinical trials, we will also pursue a number of exploratory clinical outcomes, with study coordinators collecting data daily until hospital discharge or death. The primary clinical outcome will be 
the duration of postoperative mechanical ventilation for each study participant, determined by subtracting the time of ICU admission from the time of endotracheal extubation. If the study participant is extubated prior to ICU admission, the duration of mechanical ventilation will be assigned as 0 hours. Recognising the potential for early death (intraoperative or early postoperative) biassing the primary clinical outcome, the number of ventilator-free days (VFD) at postoperative day 28 will also be determined, with those who die prior to day 28 being assigned zero VFD. Participants discharged from the hospital alive prior to day 28 will be assumed to have had no additional days of mechanical ventilation following hospital discharge. Additional secondary clinical outcomes will include evaluations of hypoxemia including oxygen saturation measured by pulse oximetry $\left(\mathrm{SpO}_{2}\right)$ and the ratio of arterial partial pressure of oxygen $\left(\mathrm{PaO}_{2}\right)$ to fraction of inspired oxygen $\left(\mathrm{FiO}_{2}\right)$, duration of oxygen supplementation, clinical diagnoses of TRALI, possible TRALI, and/ or TACO, sequential organ failure assessment (SOFA) scores, and durations of ICU and hospital stay.

\section{Sample size estimation}

The sample size for this clinical trial is based on the aforementioned mechanistic outcomes (study aim 2), with estimates of the range of effect sizes for biomarkers considered in this study derived from a previous investigation. ${ }^{45}$ Using an approximation of the SD derived from the interquartile range ( $\mathrm{IQR}$; i.e. $\mathrm{SD} \approx \mathrm{IQR} / 1.35$ ), the median effect size was found to be 0.4 , a magnitude of change that is considered relevant and appropriate to power this study. With equal allocation between groups, the sample size is estimated to be 78 participants per group. This assumes a type one error rate (alpha) $=0.10$ (two-sided) and a power of $80 \%$. Actual power is expected to be higher due to the repeated measures design. To allow for drop out and non-feasible cases, 170 total participants will be randomised with approximately 85 per treatment arm.

\section{Randomization and blinding}

Randomization in a 1:1 fashion will occur following the acquisition of signed informed consent. Randomization to the RBC washing or control group will be conducted by the study's electronic data management system's Balance (Medidata) algorithm. This software uses dynamic minimization stratified by clinical centre. The software will return a confirmation of the randomization indicating the study participant's treatment allocation status. A note will be placed into the electronic health record (EHR) identifying the patient as a study participant.

In light of the time-sensitive, point-of-care nature of the intervention, the patient, clinical team, and study team will not be blinded to the patient's treatment allocation status. Additionally, the transfusion medicine service will have unblinded, electronic access to the treatment assignment. Blinding, however, will be ensured for the physicians and laboratory personnel involved in biomarker analyses.

\section{Statistical methods}

Aim 1 of the protocol is centred on the feasibility, safety, and efficacy of POC allogeneic RBC washing. Feasibility is defined as the administration of protocol RBCs instead of off-protocol standard-issue RBCs. At the patient level, a washed arm patient is considered feasible if at least $50 \%$ of administered RBCs are washed per-protocol. Simon's optimal two-stage design will be used to determine if the protocol needs to be modified to prepare RBCs prior to the surgical procedure. The null hypothesis feasibility rate, $\mathrm{p}_{0}=0.75$, will be tested against a one-sided alternative that feasibility is higher. In the first stage, 16 patients will be accrued in the RBC washing arm with at least one RBC unit transfused on the operative day. If 12 or fewer patients were deemed feasible, the protocol will be modified to pre-wash RBCs (see limitations section of discussion for more details). If 13 or more are feasible, 32 additional patients in the RBC washing arm will be evaluated. The null hypothesis will be rejected if 40 or more of the 48 studied patients are considered feasible and the study will continue as originally planned. Else if 39 or fewer patients are deemed feasible, the protocol will be modified to pre-wash. This design yields a type I error rate of 0.10 and at least $90 \%$ power when the true patient feasibility rate is 0.90 or higher. The change in haemoglobin after the first transfused unit will be used as the primary safety measure. Additional safety endpoints including CFH and haptoglobin levels will be collected and analysed at multiple time points as described previously. Wilcoxon rank sum tests will be used to compare changes in these continuous outcome measures between randomised groups. These analyses will be conducted utilising 'as-treated' principles. Specifically, a participant who has received one or more units of CATS washed allogeneic RBC transfusion(s) on the day of surgery will be assigned and analysed as a member of the washed cohort. Those who received allogeneic RBC transfusions on the day of surgery, none of which were washed, will be assigned to the standard-issue cohort. Categorical variables (eg, development of acute kidney injury) will be assessed with chi-square tests. The change in concentrations of soluble BRMs pre- to post-wash (washed arm, washed RBC units only) will be assessed using paired t-tests or Wilcoxon signed-rank tests. To balance assay costs while ensuring scientific success, pre- and post-wash samples will be obtained and analysed for the first 75 washed RBC units. If efficacy is not clearly established, pre- and post-wash samples will be obtained for an additional 75 washed units. As leucocyte reduction methods may vary between the two clinical sites depending on the use of whole blood-derived or apheresis RBCs, sensitivity analyses will be performed stratified by clinical site.

Aim two examines the changes in RBC recipient's intermediate markers of respiratory injury or dysfunction (biomarkers) over four time points relative to the patient's first transfusion as described above. Mixed models will be fit to model the linear trajectory of these biomarkers. A model with a random slope and intercept 
will be considered initially, and the primary parameter of interest will be the treatment group by time interaction. For these analyses, an as-treated principle will be considered. Patients who receive at least one unit of washed cells will be included in the RBC washing group. Those not receiving RBC washed cells will be in the control group, as this aim is focused on mechanistic action and demonstrating biologic plausibility prior to formal evaluation of clinical outcomes, which would be analysed using traditional intention to treat (ITT) considerations. Since the functional form of the changes in biomarkers over time is not known, a discrete (3 D.F. test) representation of time will also be used to gauge the linearity assumption, as well as provide a sensitivity analysis to the primary regression model. Standard mixed modelling practices will be utilised (eg, assessment of residuals, verification of variance components, nested modelling to simply variance components and covariance patterns). This modelling scenario will be conducted for each biomarker of interest. Since prior research has noted that these outcomes are clustered, the previously described methodology by Shi et al will be used to adjust for multiple comparisons. ${ }^{46} \mathrm{We}$ will also compute O'Brien's nonparametric global test statistic to provide an overall measure of treatment effect between the two treatment groups.

Due to our desire to evaluate the impact of RBC washing in a pragmatic and clinically relevant setting, effect modification by RBC storage duration will be assessed. For each patient, separately mean and maximum RBC storage duration (among transfused RBC units) will be considered as effect modifiers using interaction terms in the above models. Similarly, total number of transfused RBC units will be considered as a potential effect modifier.

Cardiopulmonary response values are measured preand post-transfusion for each transfused unit. Linear regression with generalised estimating equations (GEE) will assess the relationship between randomised group and change in cardiopulmonary response, accounting for the correlation of observations within individuals receiving multiple transfusions.

As a final component of aim 2, we will test the hypothesis that lower levels of putative BRMs (neutral lipids, sCD40L, CCL5, RBC-MPs, CFH) in transfused RBC components (and in the RBC recipient) will be associated reduced levels of lung injury biomarkers and an attenuated cardiopulmonary response to RBC transfusion. We will specifically quantify the relationships of the putative BRMs as measured in the post-wash bag or unwashed bag (as well as in the recipient) with measures of lung injury and cardiopulmonary response. Multiple linear regression models will test for the joint effect of randomised group and the randomised group by BRM interaction term in order to determine if the relationships of BRMs with markers of lung injury and cardiopulmonary response are co-incident (similar relationship) between study groups. Validated lung-injury associated biomarkers levels are measured at four time points relative to a patient's first RBC transfusion [pre-transfusion (but after the decision to transfuse is made), within $30 \mathrm{~min}$ post-transfusion, 6 hours post, and 18 hours post (all relative to first transfusion)]. Mixed models will be fit to model the linear trajectory of these biomarkers. Cardiopulmonary response is measured before and immediately after each RBC Unit transfused; linear regression using GEE will assess this relationship.

Aim three will utilise standard analytical measures for comparing randomised treatment groups under the ITT paradigm. Continuous outcomes will be analysed using t-tests, or, for skewed data such as duration of mechanical ventilation, Wilcoxon rank sum tests will be used to compare groups. Binary outcomes will be analysed using Pearson chi-square or exact tests. Serial measurements (eg, arterial oxygen saturation) will be analysed using longitudinal summary statistics. Of note, this study is not powered for these intermediate clinical outcomes. Estimates of precision with confidence intervals along with the range of responses will be used to guide subsequent trial designs, including a larger phase II/III trial with clinical outcomes as the primary outcome of interest.

Consistent with early phase clinical trials, a higher level of significance than 0.05 is selected and we consider p-values less than 0.10 to be significant. This will facilitate advancement of the technique should it prove feasible with potential efficacy. Multiple testing may also increase the overall family wise error rate, so further research, particularly with clinical events, may be needed to quantify clinical efficacy of the approach. Missing data is expected to be minimal given the close surveillance provided in the surgical and ICU environments. However, missing specimens may occur in the event of patient discharge, death or administrative issues. Initial analyses will be conducted with the assumption of missing completely at random. Sensitivity analyses using multiple imputation and pattern mixture models will be used to assess the robustness of the model assumptions.

\section{Data quality and management}

Data quality and safety will be monitored by each site's principal investigator (PI). In addition, strategies to achieve a high level of protocol adherence will include: (1) refresher education sessions for study coordinators, (2) weekly checks of protocol compliance by the Mayo Clinic research coordinators, and (3) computerised identification of protocol violations in the database. Mayo Clinic has implemented an enterprise-wide Clinical Trials Management System (CTMS). CTMS is a data management infrastructure that operates in compliance with 21 Code of Federal Regulations (CFR) Part 11 to support multicenter clinical trials and participant registries. The core of the CTMS project is the Medidata RAVE product, which will serve as the electronic data capture and randomization system for the study. The system has comprehensive audit trails, user authentication, security and disaster plans, and standardised training for users. The system provides real-time data integrity checks, maximising data integrity while lessening the need for on-site 
source document verification. Protocol amendments will be fully vetted by the site's principal investigators (Mayo Clinic: Daryl J. Kor, MD; Duke University Medical Centre: Ian Welsby, MBBS) prior to submission for approval by each site's IRB. The investigation's final trial dataset will be available to both sites principal investigators (Mayo Clinic: Daryl J. Kor, MD; Duke University Medical Centre: Ian Welsby, MBBS). Study data will be available for secondary use by contacting the Mayo Clinic principal investigator, Dr. Daryl J. Kor, MD. Access to study data will be made available only for the subset of trial participants who have consented to the use of their study data for this purpose.

\section{Ethics and dissemination}

\section{Adverse outcomes}

Safety data including adverse events such as the development of TRALI, TACO, organ failure (including acute kidney injury), prolonged hospitalisation, ICU admission, and mortality will be recorded. Other adverse events will be monitored by the site PI and research specialist in real time from the start of randomization to hospital discharge or death. Adverse events will be defined as 'unexpected,' 'expected' and 'serious.' As our patient population is by definition 'critically ill' due to their highrisk surgical procedure, it is expected that they will have a number of unrelated adverse health events during the course of their hospital stay. Therefore, we will limit the scope of our adverse event monitoring and recording to the following:

- Serious adverse events (SAEs) will be defined as:

- Death, believed to be related to the study procedures or a death that is unexpected considering the acuity of a patient.

- A life-threatening experience believed to be related to the study procedures.

- Persistent or significant disability or incapacity that is of greater frequency or severity than what would be normally expected in the perioperative course.

- An event that jeopardises the human subject and may require medical or surgical treatment to prevent one of the preceding outcomes and is not expected in the perioperative course.

- Adverse events possibly related to the study procedures will be defined as:

- Profound anaemia (hemoglobin $<7 \mathrm{~g} / \mathrm{dL}$ ).

- Renal failure requiring renal replacement therapy.

- Myocardial infarction.

- Non-haemorrhagic stroke.

- Mesenteric ischemia requiring laparotomy (ischaemic events secondary to anaemia).

- Bloodstream infections.

Role of the data safety and monitoring board

All serious adverse events will be reported to the site institutional review board (IRB) within 24 hours of discovery followed by a more detailed written report to the IRB.
The following information about adverse events will be collected: (1) the onset and resolution of the event, (2) an assessment of the severity or intensity of the event, (3) an assessment of the relationship of the event to the intervention, and (4) any action taken because of event. Reporting of SAEs to the respective IRBs will be conducted by the PI at each site. All potentially related SAEs will be reported to the data safety monitoring board (DSMB) and to NHLBI within 7 days of discovery. Additionally, a summary report will be provided to the DSMB prior to each DSMB meeting, at least every 6 months. Safety oversight will be performed by a DSMB, whose members will be independent from the study investigators. Safety endpoints consisting of expected clinical events, including death, will be assessed for all participants who are enrolled in the study on an intent-to-treat basis. Safety endpoints, as well as all serious and unexpected adverse events, will be summarised by treatment group. Trial conduct will be audited by the DSMB at least every 6 months.

\section{Ethics approval}

Prior to enrollment of the first study participant, protocol approval was obtained from the DSMB, each participating institutional IRB, and the NHLBI. Compliance of informed consent forms with NHLBI requirements and the CFRs Title 21 Part 50 Section 50.25 was ensured. Documentation of all IRB approvals, including all finalised consent forms, have been collected and stored by the study team.

\section{Considerations for continuation to a phase II/III clinical trial}

This phase I/II clinical trial is not powered to detect subtle differences in clinical outcomes, which would be more adequately addressed in a much larger phase II/ III clinical trial. Nonetheless, the clinical evaluations outlined in this protocol will provide essential preliminary data that can inform the merit and feasibility of a future phase II/III clinical trial. Moreover, if POC RBC-washing is determined not to be feasible, safe, or efficacious (aim 1 ), then this would provide evidence against pursuit of a larger clinical trial. Additionally, if no substantial impact is seen in the intermediate markers of respiratory injury/ dysfunction (aim 2), there would be limited benefit in pursuing a larger trial.

\section{Protocol amendments}

Protocol amendments will be fully vetted by the site's principal investigators (Mayo Clinic: Daryl J. Kor, MD; Duke University Medical Centre: Ian Welsby, MBBS) prior to submission for approval by each site's IRB.

\section{Access to data}

The investigation's final trial dataset will be available to both sites principal investigators (Mayo Clinic: Daryl J. Kor, MD; Duke University Medical Centre: Ian Welsby, MBBS). Study data will be available for secondary use by contacting the Mayo Clinic principal investigator, Dr. Daryl J. Kor, MD. Access to study data will be made 
available only for the subset of trial participants who have consented to the use of their study data for this purpose.

\section{Dissemination policy}

Study findings, including those of associated ancillary studies, will be disseminated to the scientific community in abstract and oral presentation formats at major national and international medical specialty meetings. All published manuscripts will be submitted to Pub Med Central in accordance with the National Institute of Health Public Access Policy.

\section{Ancillary studies}

Ancillary study proposals that complement or advance the specific proposals of this study protocol will be encouraged. Proposals will be reviewed by the Co-PIs of this protocol (Drs. Daryl Kor and Ian Welsby), both to ensure scientific merit and validity as well as ensuring consistency with the goals and conduct of the main study. Such ancillary studies may utilise data and/or samples accrued during the clinical trial or, when feasible, additional data may be collected. All statistical plans will be reviewed a priori and approved before data analysis is initiated. All presentations and manuscripts will require explicit review and approval by this investigation's Co-PIs.

\section{Protocol funding}

This study is supported by the NIH-NHLBI (Grant Number: R01 HL121232), the Mayo Clinic Critical Care and Anesthesiology and Perioperative Medicine Research Committees, as well as the Duke Clinical Anaesthesia Research Endeavours (CARE). Funding and time allotment has been provided by each of these entities to support study personnel, protocol development and data management (Medidata Rave), sample acquisition, transfusion procedures, sample and data processing and storage, and statistical support. There is no influence exerted by funding sources on the scientific conduct of the study protocol including data collection, analyses, or interpretation. Additionally, funding sources will play no role in the preparation of study results for presentation or publication.

\section{DISCUSSION}

Strategies that may decrease the incidence of RBC-associated pulmonary complications, particularly those that can be rapidly disseminated to clinical practice, remain undefined. We have presented the study protocol and data analysis plans for a phase I/II, multicenter, randomised clinical trial that seeks to test the feasibility, safety, and efficacy of POC washing of allogeneic RBCs in cardiac surgery with the goal of attenuating transfusion-related pulmonary complications. Specifically, we hypothesise that POC washing of allogeneic RBCs in cardiac surgery patients will be feasible, safe, and efficacious for the removal of soluble BRMs. Additionally, we hope to gain important mechanistic information regarding the relationship between these potentially pathogenic BRMs and intermediate markers of both TRALI (lung injury biomarkers) and TACO (cardiopulmonary physiologic indices) in transfused patients undergoing cardiovascular surgery. Finally, important clinical outcomes will also be assessed in order to provide essential information in determining the value and feasibility of a larger phase II/ III clinical trial of RBC-washing for the reduction of transfusion-related pulmonary complications.

\section{Limitations}

Despite notable strengths of this study protocol including a large and accessible at-risk population, an established clinical trial infrastructure, and multidisciplinary experience and expertise in translational, patient-centred transfusion research, there are also limitations. The first relates to the feasibility of point-of-care RBC washing in a time-sensitive environment such as cardiac surgery. Though experience regarding the feasibility of washing allogeneic RBC units in this patient environment is limited, both centres have substantial experience with the successful implementation of autotransfusion practices. This will be invaluable in ensuring the feasibility of the study protocol. In the unlikely circumstance that feasibility becomes a barrier to progress, we will perform pre-washing of two units of allogeneic RBCs at the time of OR entry for those adjudicated to the washing arm of the trial. These pre-washed RBC units would be stored in appropriate blood coolers until the time of RBC need is determined by the clinical team. Of note, washed RBCs can be stored in coolers for up to 18 hours, as the coolers have been validated to maintain a temperature range between 1 and 6 degrees Centigrade for this length of time. All subsequent units could then be washed as described above. Of note, storage of allogeneic RBCs in blood coolers in the OR for the duration of the surgical procedure is standard of care at the two participating institutions for patients who are predicted at high-risk for RBC transfusion. It should be noted the proposed design to evaluate feasibility of real-time washing preserves the blood product supply and minimises waste in the event RBCs are not required by the patient.

As a second limitation, our candidate biomarkers may not represent or capture true causal pathways. If promising alternative biomarkers and mechanistic pathways are identified, our stored blood samples from this investigation will be available for future analyses for all study participants providing consent for the use of their specimens for this purpose. Additionally, while we are measuring the concentrations of relevant BRMs from both the RBC unit and the transfusion recipient, we are not measuring the hematocrit of the RBC unit, which may result in incomplete characterisation of the total dose of transfused BRMs. Importantly, it is also possible that the putative agents are the RBCs themselves rather than contaminants of the RBC supernatant. Indeed, if washed RBC transfusions show no impact on recipient responses, this may in fact support a key role for the RBC itself rather than BRMs in the RBC storage supernatant. Although an unexpected finding, this would provide essential insight 
guiding future research on mitigating RBC-associated TRALI and TACO.

A third concern is that the inflammatory response seen in cardiac surgery may mask between-group differences in our analyses. Previous evaluations of patients undergoing cardiac surgery have identified a significant increase in IL-6 concentrations in those who receive RBC transfusion versus those who do not. ${ }^{47}$ However, IL-6 concentrations following cardiac surgery have been shown to remain under $200 \mathrm{pg} / \mathrm{mL} .{ }^{48}{ }^{49}$ This concentration falls well below levels typically encountered with lung injury, which are frequently greater than $500 \mathrm{pg} / \mathrm{mL}^{45}{ }^{50}$ Additionally, we expect an even greater separation of biomarkers evaluating specific lung-injury pathways rather than markers of general inflammation.

Another area of concern relates to RBC storage duration. Although equipoise remains, clinical data suggests the potential importance of RBC storage duration on patient-important outcomes. ${ }^{51-54}$ Previous work has also shown clear temporal changes in the biochemical profile of stored RBC supernatant. ${ }^{19212255-57}$ Recent evidence suggests that RBC storage age beyond 6 weeks results in increased extravascular hemolysis but storage age of 5 weeks or less does not. ${ }^{58}$ Therefore, it is possible that variability in RBC storage duration may impact our results. However, we have outlined a statistical plan to address this potential concern (see statistical considerations). In addition, we hypothesise that the washing protocol will attenuate the effects of storage duration. It should also be mentioned that washed RBC units outdate after 24 hours. Hence, any washed RBCs not transfused within 24 hours will be discarded and the incidence of this occurrence recorded. However, as washed RBCs will only be administered on the day of surgery, this should not impact study results.

Finally, the study protocol is not adequately powered to fully evaluate clinical outcomes (Aim 3). Such hypotheses would more adequately be addressed in a larger phase II/III clinical trial. Nonetheless, we believe the clinical evaluations outlined in this protocol will provide essential preliminary data that can inform the merit and feasibility of a future more definitive phase II/III clinical trial.

\section{Author affiliations}

${ }^{1}$ Division of Critical Care, Department of Anesthesiology and Perioperative Medicine, Mayo Clinic Minnesota, Rochester, Minnesota, USA

${ }^{2}$ Department of Anesthesiology, Duke University Medical Center, Raleigh, North Carolina, USA

${ }^{3}$ Blood Systems Research Institute,University of California, San Francisco, California, USA

${ }^{4}$ Bonfils Blood Center, University of Colorado Denver, Denver, Colorado, USA

${ }^{5}$ Department of Anesthesiology and Perioperative Medicine, Mayo Clinic, Rochester, Minnesota, USA

${ }^{6}$ Department of Laboratory Medicine and Pathology, Mayo Clinic, Rochester,

Minnesota, USA

${ }^{7}$ Department of Biomedical Statistics and Informatics, Mayo Clinic, Rochester,

Minnesota, USA

${ }^{8}$ Department of Anesthesiology, Duke University Medical Center, Raleigh, North Carolina, USA

${ }^{9}$ Laboratory Medicine and Pathology, Mayo Clinic, Rochester, MN
Acknowledgements The authors would like to acknowledge Dr. Sheila Keating, $\mathrm{PhD}$ (Blood Systems Research Institute, San Francisco, CA) for her diligent assistance with laboratory analyses and biomarker handling.

Contributors MAW. Contributed to the study design and conduct, and writing of the manuscript.lan JW Contributed to the conception of the work, study design and conduct, acquisition and interpretation of data, and writing of the manuscript. PN. Contributed to the study design and conduct, acquisition and interpretation of data, and writing of the manuscript.CS, Contributed to the study design and conduct, and writing of the manuscript.SA. Contributed to the study design and conduct, and writing of the manuscript.EW. Contributed to the study design and conduct, and writing of the manuscript.Paula J. Santrach, MD. Contributed to the study design and conduct, and writing of the manuscript.LM, RN. Contributed to the study design and conduct, acquisition and interpretation of data, and writing of the manuscript.LL, RRT. Contributed to the study design and conduct, acquisition and interpretation of data, and writing of the manuscript.CN, MLS(ASCP). Contributed to the study design and conduct, acquisition and interpretation of data, and writing of the manuscript.BD, RN. Contributed to the study design and conduct, acquisition and interpretation of data, and writing of the manuscript.CVB. Contributed to the study design and conduct, and writing of the manuscript.PS. Contributed to the study design and conduct, acquisition and interpretation of data, and writing of the manuscript.REC. Contributed to the conception of the work, study design and conduct, acquisition and interpretation of data, and writing of the manuscript. DJK. Contributed to the conception of the work, study design and conduct, acquisition and interpretation of data, and writing of the manuscript.All co-authors have provided final approval of the current manuscript version and agree to be accountable for all aspects of the work in ensuring that questions related to the accuracy or integrity of any part of the work are appropriately investigated and resolved.

Funding This study was made possible by funding from the Mayo Clinic Department of Anesthesiology and Perioperative Medicine andthe Critical Care Integrated Multidisciplinary Practice,Rochester, Minnesota. In addition, this study was supported by an NIH grant number: R01 HL121232, PI: Dr. Kor. The funding sources had no role in the study design, collection, management, analysis, and interpretation of data, writing of this report, nor decision to submit this report for publication.

\section{Competing interests None declared.}

Patient consent Study specific written consent forms (please see supplemental materials) will be signed by each patient prior to enrollment. Compliance of informed consent forms with NHLBI requirements and the CFRs Title 21 Part 50 Section 50.25 was ensured. Documentation of all IRB approvals, including all finalized consent forms, have been collected and stored by the study team.

Ethics approval Prior to enrollment of the first study participant, protocol approval was obtained from the DSMB, each participating institutional IRB, and the NHLBI.

Provenance and peer review Not commissioned; externally peer reviewed.

Data sharing statement At this stage of the study (protocol), no unpublished data is available. The investigation's final trial dataset will be available to both sites principal investigators (Mayo Clinic: Daryl J. Kor, MD; Duke University Medical Center: Ian Welsby, MBBS). Study data will be available for secondary use by contacting the Mayo Clinic principal investigator, Dr. Daryl J. Kor, MD. Access to study data will be made available only for the subset of trial participants who have consented to the use of their study data for this purpose.

Open Access This is an Open Access article distributed in accordance with the Creative Commons Attribution Non Commercial (CC BY-NC 4.0) license, which permits others to distribute, remix, adapt, build upon this work non-commercially, and license their derivative works on different terms, provided the original work is properly cited and the use is non-commercial. See: http://creativecommons.org/ licenses/by-nc/4.0/

(c) Article author(s) (or their employer(s) unless otherwise stated in the text of the article) 2017. All rights reserved. No commercial use is permitted unless otherwise expressly granted.

\section{REFERENCES}

1. Vlaar AP, Binnekade JM, Prins D, et al. Risk factors and outcome of transfusion-related acute lung injury in the critically ill: a nested casecontrol study. Crit Care Med 2010;38:771-8. 
2. Gajic O, Rana R, Winters JL, et al. Transfusion-related acute lung injury in the critically ill: prospective nested case-control study. Am J Respir Crit Care Med 2007;176:886-91.

3. Silliman CC, Fung YL, Ball JB, et al. Transfusion-related acute lung injury (TRALI): current concepts and misconceptions. Blood Rev 2009;23:245-55.

4. Popovsky MA. The Emily Cooley Lecture 2009 to breathe or not to breathe-that is the question. Transfusion 2010:2057-62.

5. Popovsky MA. Transfusion and the lung: circulatory overload and acute lung injury. Vox Sang 2004;87:62-5.

6. Li G, Kojicic M, Reriani MK, et al. Long-term survival and quality of life after transfusion-associated pulmonary edema in critically ill medical patients. Chest 2010;137:783-9.

7. Bux J, Sachs UJ. The pathogenesis of transfusion-related acute lung injury (TRALI). Br J Haematol 2007;136:788-99.

8. Rana R, Fernández-Pérez ER, Khan SA, et al. Transfusion-related acute lung injury and pulmonary edema in critically ill patients: a retrospective study. Transfusion 2006;46:1478-83.

9. Kopko PM, Popovsky MA. Pulmonary injury from transfusion-related acute lung injury. Clin Chest Med 2004;25:105-11.

10. Silliman CC, Moore EE, Kelher MR, et al. Identification of lipids that accumulate during the routine storage of prestorage leukoreduced red blood cells and cause acute lung injury. Transfusion 2011;51:2549-54.

11. Silliman CC. The two-event model of transfusion-related acute lung injury. Crit Care Med 2006;34:S124-S131.

12. Khan SY, Kelher MR, Heal JM, et al. Soluble CD40 ligand accumulates in stored blood components, primes neutrophils through CD40, and is a potential cofactor in the development of transfusion-related acute lung injury. Blood 2006;108:2455-62.

13. Bierbaum BE, Callaghan JJ, Galante JO, et al. An analysis of blood management in patients having a total hip or knee arthroplasty. $J$ Bone Joint Surg Am 1999;81:2-10.

14. Popovsky MA, Audet AM, Andrzejewski C. Transfusion-associated circulatory overload in orthopedic surgery patients: a multiinstitutional study. Immunohematology 1996;12:87-9.

15. Bless NM, Huber-Lang M, Guo RF, et al. Role of CC chemokines (macrophage inflammatory protein-1 beta, monocyte chemoattractant protein-1, RANTES) in acute lung injury in rats. $J$ Immunol 2000;164:2650-9.

16. Andrzejewski C, Popovsky MA, Stec TC, et al. Hemotherapy bedside biovigilance involving vital sign values and characteristics of patients with suspected transfusion reactions associated with fluid challenges: can some cases of transfusion-associated circulatory overload have proinflammatory aspects? Transfusion 2012;52:2310-20.

17. Klein HG, Anstee DJ, eds. Mollison's Blood Transfusion in Clinical Medicine. 11 ed. Malden, MA: Oxford: Blackwell Publishing, 2006.

18. Vermeulen Windsant IC, de Wit NC, Sertorio JT, et al. Blood transfusions increase circulating plasma free hemoglobin levels and plasma nitric oxide consumption: a prospective observational pilot study. Crit Care 2012;16:R95.

19. Stapley R, Owusu BY, Brandon A, et al. Erythrocyte storage increases rates of $\mathrm{NO}$ and nitrite scavenging: implications for transfusion-related toxicity. Biochem J 2012;446:499-508.

20. Singel DJ, Stamler JS. Chemical physiology of blood flow regulation by red blood cells: the role of nitric oxide and S-nitrosohemoglobin. Annu Rev Physiol 2005;67:99-145.

21. Jy W, Ricci M, Shariatmadar S, et al. Microparticles in stored red blood cells as potential mediators of transfusion complications. Transfusion 2011;51:886-93.

22. Gladwin MT, Kim-Shapiro DB. Storage lesion in banked blood due to hemolysis-dependent disruption of nitric oxide homeostasis. Curr Opin Hematol 2009;16:515-23.

23. Burnier L, Fontana P, Kwak BR, et al. Cell-derived microparticles in haemostasis and vascular medicine. Thromb Haemost 2009;101:439-51.

24. Westphal-Varghese B, Erren M, Westphal M, et al. Processing of stored packed red blood cells using autotransfusion devices decreases potassium and microaggregates: a prospective, randomized, single-blinded in vitro study. Transfus Med 2007;17:89-95.

25. Rosolski T, Matthey T, Frick U, et al. Blood separation with two different autotransfusion devices: effects on blood cell quality and coagulation variables. Int J Artif Organs 1998;21:820-4.

26. Gruber M, Breu A, Frauendorf M, et al. Washing of banked blood by three different blood salvage devices. Transfusion 2013;53:1001-9.

27. Booke $M$, Fobker M, Fingerhut $D$, et al. Fat elimination during intraoperative autotransfusion: an in vitro investigation. Anesth Analg 1997;85:959-62.
28. Blumberg N, Heal JM, Gettings KF, et al. An association between decreased cardiopulmonary complications (transfusion-related acute lung injury and transfusion-associated circulatory overload) and implementation of universal leukoreduction of blood transfusions. Transfusion 2010;50:2738-44.

29. Blumberg N, Heal JM, Rowe JM. A randomized trial of washed red blood cell and platelet transfusions in adult acute leukemia [ISRCTN76536440]. BMC Blood Disord 2004;4:6.

30. Biffl WL, Moore EE, Offner PJ, et al. Plasma from aged stored red blood cells delays neutrophil apoptosis and primes for cytotoxicity: abrogation by poststorage washing but not prestorage leukoreduction. J Trauma 2001;50:426-32. discussion 32.

31. Silliman CC, Moore EE, Johnson JL, et al. Transfusion of the injured patient: proceed with caution. Shock 2004;21:291-9.

32. The 2011 National blood collection and utilization survey. Washington, DC: Department of Health and Human Services, 2011.

33. Reeves BC, Murphy GJ. Increased mortality, morbidity, and cost associated with red blood cell transfusion after cardiac surgery. Curr Opin Cardiol 2008;23:607-12.

34. Koch CG, Li L, Sessler DI, et al. Duration of red-cell storage and complications after cardiac surgery. $N$ Engl $J$ Med 2008;358:1229-39.

35. Koch CG, Li L, Duncan Al, et al. Morbidity and mortality risk associated with red blood cell and blood-component transfusion in isolated coronary artery bypass grafting. Crit Care Med 2006;34:1608-16.

36. Koch C, Li L, Figueroa P, et al. Transfusion and pulmonary morbidity after cardiac surgery. Ann Thorac Surg 2009;88:1410-8.

37. Goudie R, Sterne JA, Verheyden V, et al. Risk scores to facilitate preoperative prediction of transfusion and large volume blood transfusion associated with adult cardiac surgery. $\mathrm{Br} J$ Anaesth 2015;114:757-66.

38. Murphy GJ, Reeves BC, Rogers CA, et al. Increased mortality, postoperative morbidity, and cost after red blood cell transfusion in patients having cardiac surgery. Circulation 2007;116:2544-52.

39. Li G, Rachmale S, Kojicic M, et al. Incidence and transfusion risk factors for transfusion-associated circulatory overload among medical intensive care unit patients. Transfusion 2011;51:338-43.

40. O'Leary MF, Szklarski P, Klein TM, et al. Hemolysis of red blood cells after cell washing with different automated technologies: clinical implications in a neonatal cardiac surgery population. Transfusion 2011;51:955-60.

41. Mehta RL, Kellum JA, Shah SV, et al. Acute Kidney Injury Network: report of an initiative to improve outcomes in acute kidney injury. Crit Care 2007;11:R31.

42. Bakkour S, Acker JP, Chafets DM, et al. Manufacturing method affects mitochondrial DNA release and extracellular vesicle composition in stored red blood cells. Vox Sang 2016;111:22-32.

43. Danesh A, Inglis HC, Jackman RP, et al. Exosomes from red blood cell units bind to monocytes and induce proinflammatory cytokines, boosting T-cell responses in vitro. Blood 2014;123:687-96.

44. Bridges EJ, Woods SL. Pulmonary artery pressure measurement: state of the art. Heart Lung 1993;22:99-111.

45. Fremont RD, Koyama T, Calfee CS, et al. Acute lung injury in patients with traumatic injuries: utility of a panel of biomarkers for diagnosis and pathogenesis. J Trauma 2010;68:1121-7.

46. Shi Q, Pavey ES, Carter RE. Bonferroni-based correction factor for multiple, correlated endpoints. Pharm Stat 2012;11:300-9.

47. Fransen E, Maessen J, Dentener M, et al. Impact of blood transfusions on inflammatory mediator release in patients undergoing cardiac surgery. Chest 1999:116:1233-9.

48. Hirai S. Systemic inflammatory response syndrome after cardiac surgery under cardiopulmonary bypass. Ann Thorac Cardiovasc Surg 2003;9:365-70.

49. Allan CK, Newburger JW, McGrath E, et al. The relationship between inflammatory activation and clinical outcome after infant cardiopulmonary bypass. Anesth Analg 2010;111:1244-51.

50. Meduri GU, Headley S, Kohler G, et al. Persistent elevation of inflammatory cytokines predicts a poor outcome in ARDS. plasma IL-1 beta and IL- 6 levels are consistent and efficient predictors of outcome over time. Chest 1995;107:1062-73.

51. Koch CG, Li L, Sessler DI, et al. Duration of red-cell storage and complications after cardiac surgery. $N$ Engl $J$ Med 2008;358:1229-39.

52. Eikelboom JW, Cook RJ, Liu Y, et al. Duration of red cell storage before transfusion and in-hospital mortality. Am Heart $J$ 2010;159:737-43.

53. Manlhiot C, McCrindle BW, Menjak IB, et al. Longer blood storage is associated with suboptimal outcomes in high-risk pediatric cardiac surgery. Ann Thorac Surg 2012;93:1563-9. 
54. Basran S, Frumento RJ, Cohen A, et al. The association between duration of storage of transfused red blood cells and morbidity and mortality after reoperative cardiac surgery. Anesth Analg 2006;103:15-20. table of contents.

55. Silliman CC, Clay KL, Thurman GW, et al. Partial characterization of lipids that develop during the routine storage of blood and prime the neutrophil NADPH oxidase. J Lab Clin Med 1994:124:684-94.

56. Kristiansson M, Soop M, Saraste L, et al. Cytokines in stored red blood cell concentrates: promoters of systemic inflammation and simulators of acute transfusion reactions? Acta Anaesthesiol Scand 1996;40:496-501.

57. Nielsen HJ, Reimert CM, Pedersen AN, et al. Time-dependent, spontaneous release of white cell- and platelet-derived bioactive substances from stored human blood. Transfusion 1996;36:960-5.

58. Rapido F, Brittenham GM, Bandyopadhyay S, et al. Prolonged red cell storage before transfusion increases extravascular hemolysis. $J$ Clin Invest 2017;127:375-82.

59. Ware LB, Koyama T, Billheimer DD, et al. Prognostic and pathogenetic value of combining clinical and biochemical indices in patients with acute lung injury. Chest 2010;137:288-96.

60. Parsons PE, Eisner MD, Thompson BT, et al. Lower tidal volume ventilation and plasma cytokine markers of inflammation in patients with acute lung injury. Crit Care Med 2005;33:1-6. discussion 2302.

61. McClintock D, Zhuo H, Wickersham N, et al. Biomarkers of inflammation, coagulation and fibrinolysis predict mortality in acute lung injury. Crit Care 2008;12:R41.

62. Ware LB, Matthay MA, Parsons PE, et al. Pathogenetic and prognostic significance of altered coagulation and fibrinolysis in acute lung injury/acute respiratory distress syndrome. Crit Care Med 2007:35:1821-8.

63. Prabhakaran P, Ware LB, White KE, et al. Elevated levels of plasminogen activator inhibitor-1 in pulmonary edema fluid are associated with mortality in acute lung injury. Am J Physiol Lung Cell Mol Physiol 2003;285:L20-L28.

64. Christie JD, Robinson N, Ware LB, et al. Association of protein C and type 1 plasminogen activator inhibitor with primary graft dysfunction. Am J Respir Crit Care Med 2007;175:69-74.

65. Ware LB, Koyama T, Billheimer DD, et al. Prognostic and pathogenetic value of combining clinical and biochemical indices in patients with acute lung injury. Chest 2010;137:288-96.

66. Fremont RD, Koyama T, Calfee CS, et al. Acute lung injury in patients with traumatic injuries: utility of a panel of biomarkers for diagnosis and pathogenesis. J Trauma 2010;68:1121-7.

67. Ware LB, Eisner MD, Thompson BT, et al. Significance of von Willebrand factor in septic and nonseptic patients with acute lung injury. Am J Respir Crit Care Med 2004;170:766-72.

68. Ware LB, Conner ER, Matthay MA. Von Willebrand factor antigen is an independent marker of poor outcome in patients with early acute lung injury. Crit Care Med 2001;29:2325-31.
69. Rubin DB, Wiener-Kronish JP, Murray JF, et al. Elevated von Willebrand factor antigen is an early plasma predictor of acute lung injury in nonpulmonary sepsis syndrome. J Clin Invest 1990;86:474-80.

70. Moss M, Ackerson L, Gillespie MK, et al. von Willebrand factor antigen levels are not predictive for the adult respiratory distress syndrome. Am J Respir Crit Care Med 1995;151:15-20.

71. Calfee CS, Eisner MD, Parsons PE, et al. Soluble intercellular adhesion molecule-1 and clinical outcomes in patients with acute lung injury. Intensive Care Med 2009;35:248-57.

72. Covarrubias M, Ware LB, Kawut SM, et al. Plasma intercellular adhesion molecule-1 and Von Willebrand factor in primary graft dysfunction after lung transplantation. Am J Transplant 2007;7:2573-8

73. Agouridakis $P$, Kyriakou D, Alexandrakis MG, et al. The predictive role of serum and bronchoalveolar lavage cytokines and adhesion molecules for acute respiratory distress syndrome development and outcome. Respir Res 2002;3:25.

74. Eisner MD, Parsons P, Matthay MA, et al. Plasma surfactant protein levels and clinical outcomes in patients with acute lung injury. Thorax 2003;58:983-8.

75. Greene KE, Wright JR, Steinberg KP, et al. Serial changes in surfactant-associated proteins in lung and serum before and after onset of ARDS. Am J Respir Crit Care Med 1999;160:1843-50.

76. Christie JD, Shah CV, Kawut SM, et al. Plasma levels of receptor for advanced glycation end products, blood transfusion, and risk of primary graft dysfunction. Am J Respir Crit Care Med 2009;180:1010-5.

77. Calfee CS, Ware LB, Eisner MD, et al. Plasma receptor for advanced glycation end products and clinical outcomes in acute lung injury. Thorax 2008;63:1083-9.

78. Grommes J, Alard JE, Drechsler M, et al. Disruption of plateletderived chemokine heteromers prevents neutrophil extravasation in acute lung injury. Am J Respir Crit Care Med 2012;185:628-36.

79. Conti P, DiGioacchino M. MCP-1 and RANTES are mediators of acute and chronic inflammation. Allergy Asthma Proc 2001;22:133-7.

80. Donadee C, Raat NJ, Kanias T, et al. Nitric oxide scavenging by red blood cell microparticles and cell-free hemoglobin as a mechanism for the red cell storage lesion. Circulation 2011;124:465-76.

81. Li G, Daniels CE, Kojicic M, et al. The accuracy of natriuretic peptides (brain natriuretic peptide and $\mathrm{N}$-terminal pro-brain natriuretic) in the differentiation between transfusion-related acute lung injury and transfusion-related circulatory overload in the critically ill. Transfusion 2009;49:13-20.

82. Tobian AA, Sokoll LJ, Tisch DJ, et al. N-terminal pro-brain natriuretic peptide is a useful diagnostic marker for transfusion-associated circulatory overload. Transfusion 2008;48:1143-50.

83. Zhou L, Giacherio D, Cooling L, et al. Use of B-natriuretic peptide as a diagnostic marker in the differential diagnosis of transfusionassociated circulatory overload. Transfusion 2005;45:1056-63. 